\title{
WAVELET-BASED SHIFT-INVARIANT NOISE MODEL
}

\section{Fu-Tai Wang}

Department of Electrical Engineering, National Taiwan Ocean University, Keelung, Taiwan 202, R.O.C. Department of Electrical Engineering, Hwa Hsia Institute of Technology, Chung Ho, Taipei, Taiwan 235, R.O.C.

Jenny Chih-Yu Lee

Department of Microelectronic Engineering, National Kaohsiung Marine University, Nantzu, Kaohsiung, Taiwan 811, R.O.C.

Shun-Hsyung Chang

Department of Electrical Engineering, National Taiwan Ocean University, Keelung, Taiwan 202, R.O.C. Department of Microelectronic Engineering, National Kaohsiung Marine University, Nantzu, Kaohsiung, Taiwan 811, R.O.C., shchang@mail.nkmu.edu.tw

Follow this and additional works at: https://jmstt.ntou.edu.tw/journal

Part of the Electrical and Computer Engineering Commons

\section{Recommended Citation}

Wang, Fu-Tai; Lee, Jenny Chih-Yu; and Chang, Shun-Hsyung (2006) "WAVELET-BASED SHIFT-INVARIANT NOISE MODEL," Journal of Marine Science and Technology. Vol. 14: Iss. 4, Article 1.

DOI: $10.51400 / 2709-6998.2080$

Available at: https://jmstt.ntou.edu.tw/journal/vol14/iss4/1

This Research Article is brought to you for free and open access by Journal of Marine Science and Technology. It has been accepted for inclusion in Journal of Marine Science and Technology by an authorized editor of Journal of Marine Science and Technology. 


\section{WAVELET-BASED SHIFT-INVARIANT NOISE MODEL}

Acknowledgements

This work was supported by the National Science Council of the R.O.C. under contract NSC 93-2611-E019-024. 


\title{
WAVELET-BASED SHIFT-INVARIANT NOISE MODEL
}

\author{
Fu-Tai Wang*,**, Jenny Chih-Yu Lee***, and Shun-Hsyung Chang*,***
}

Key words: dual-tree discrete wavelet transform, underwater sound, detection.

\begin{abstract}
This paper proposes the use of shift-invariant dual-tree discrete wavelet transform (DT DWT) noise model to the problem of a multipath signal detection in underwater environment. The design procedure for this adaptive model of the background noise is described. When an input signal is shifted, as in a multipath environment, this shift-invariant DT DWT can generate multi-resolution subspaces. Each of them keeps more coefficient energy of this signal than DWT's. The result of computer simulations shows that this method can improve the performance of a multipath signal detection, by reducing the false alarm probability in a range of different signal-tonoise (SNR) ratios.
\end{abstract}

\section{INTRODUCTION}

Various approaches have been used for underwater signal detection by utilizing transformations of the raw data, such as the Fourier transform. Although this transform is useful, it has difficulties in analyzing shortterm transient signals. Several time-frequency approaches have been developed to address this problem. Some of researchers have tried to use wavelets to deal with it [1-3]. The wavelet transform uses long windows at low frequencies and short windows at high frequencies. When the wavelet analysis is viewed as a filter bank, the analysis filters are regularly spread in a logarithmic scale over the frequency axis. So the frequency resolution becomes arbitrarily good at low frequency, while the time resolution becomes arbitrarily good at high frequency. This kind of analysis is suitable for the signal which is composed of low frequency components of long duration and high frequency components of short duration.

Paper Submitted 10/06/05, Accepted 01/03/06. Author for Correspondence: Shun-Hsyung Chang. E-mail: shchang@mail.nkmu.edu.tw.

*Department of Electrical Engineering, National Taiwan Ocean University, Keelung, Taiwan 202, R.O.C.

**Department of Electrical Engineering, Hwa Hsia Institute of Technology, Chung Ho, Taipei, Taiwan 235, R.O.C.

***Department of Microelectronic Engineering, National Kaohsiung Marine University, Nantzu, Kaohsiung, Taiwan 811, R.O.C.
Our primary interest here is in the determination of where particular signals appear in underwater. Many approaches involve predefined models that are designed for particular situations. Instead of imposing a priori model, the authors of [1] approached the problem from an alternative means. They used an empirical model of the noise for identifying the signals. In this article we attempt to build a shift-invariant and adaptive noise model of the background continuum. Observations considered to be outliers from this noise model at any time are then flagged as potential signals. For the application in a multipath environment that contends with delays of a signal, shift-invariant DT DWT can generate multi-resolution subspaces that keep more of their coefficient energy as the input signal is shifted [2]. These retaining coefficient energy at different scales enable this method to improve the detecting ability in a range of different SNR ratios.

\section{SHIFT-INVARIANT DT-DWT NOISE MODEL}

Wavelets that are compactly supported in time domain are good for processing transient signals. Using scales and positions based on powers of two, discrete wavelet transform is much more efficient in calculations. One commonly used series of mother wavelets was constructed by Daubechies [4]. An efficient algorithm using filters to implement this scheme was obtained by Mallat [7]. This very practical filtering scheme yields a fast wavelet transform. In wavelet analysis, the lowfrequency, high-scale components of the signal are referred as the approximations and the high-frequency, low-scale contents are indicated as the details.

Some researchers have proposed using two wavelet transforms for signal analysis, such as letting one wavelet be nearly the Hilbert transforms of the other. Kingsbury found that when the lowpass filters of one DWT are offset from the lowpass filters of the second DWT by a half sample, the dual-tree DWT is nearly shift-invariant [6]. In paper [8], Selesnick gave an alternative derivation for the result by Kingsbury. Based on the limit functions defined by the infinite product formula, Selesnick designed a Hilbert transform pairs of wavelet bases and arrived at the same condition. In 
order to build an adaptive noise model of the background continuum for the application in a multipath environment, this shift-invariant dual-tree DWT is considered. The detailed procedure of designing the DT DWT can be found in [9].

In the DWT, the lowpass filter $h_{0}(n)$ fully determines the orthogonal wavelet base. According to article [9], let $h_{0}(n)$ and $h_{1}(n)$ be a class of exactly reconstructing filters called "conjugate quadrature filter" or CQF's. That is

$$
\sum_{n} h_{0}(n) h_{0}(n+2 k)=\delta(k)= \begin{cases}1, & k=0 \\ 0, & k \neq 0\end{cases}
$$

and $h_{1}(n)=(-1)^{(n)} h_{0}(M-n)$. Let $g_{0}(n)$ and $g_{1}(n)$ be a second CQF pair and assume filters $h_{i}(n)$ and $g_{i}(n)$, for $i=1$ and 2 , are real-valued. With $h_{1}(n)$ and $g_{1}(n)$, wavelet $\psi_{h}(n)$ and the other wavelet $\psi_{g}(n)$ can be defined. It was shown that if $g_{0}(n)$ is a half-sample delayed version of $h_{0}(n)$

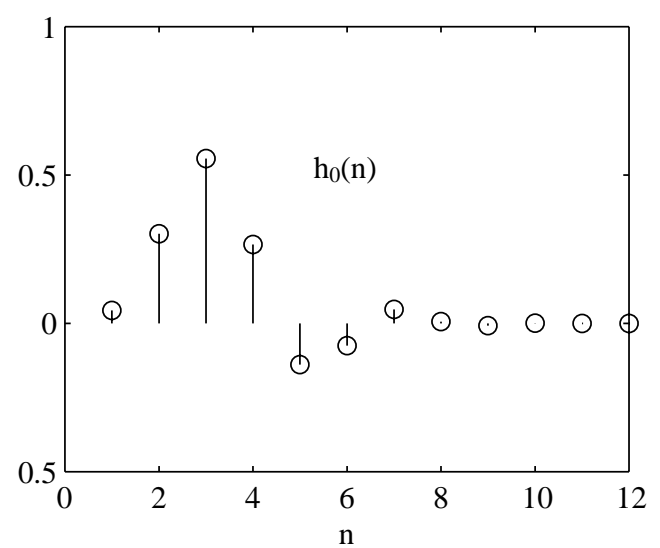

(a) Lowpass scaling filter $h_{0}(n)$

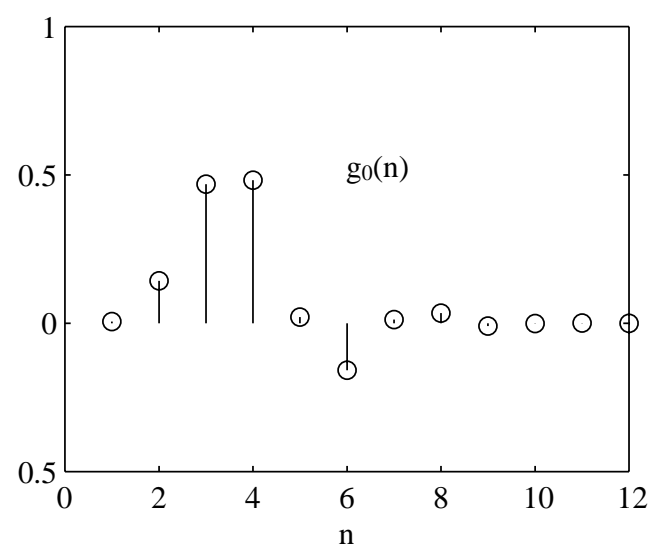

(b) Lowpass scaling filter $\mathrm{g}_{0}(\mathrm{n})$

$$
g_{0}(n)=h_{0}(n-1 / 2)
$$

then the two wavelets generated by the digital filters $h_{1}$ and $g_{1}$ respectively, form a Hilbert transform pair. Though a half-sample delay cannot be designed by a finite impulse response (FIR) filter, it can be done by using an allpass filter with approximately constant fractional delay [8]. From the designing procedure in [8], one of several pairs of digital filters $h_{0}$ and $g_{0}$ can be found. An example of them are illustrated in Figure 1 (a), (b). The plot of $\left|H_{0}(\omega)\right|$ and $\left|G_{0}(\omega)\right|$ in Figure 1 (c) shows that they are indistinguishable. And the phase response of $G_{0}(\omega) / H_{0}(\omega)$ in Figure $1(\mathrm{~d})$ shows its agreement with $\omega / 2$ near $\omega=0$.

DWT produces a hierarchically structured decomposition. The choice of a suitable level for the hierarchy depends on the signal. At each level $\mathrm{j}$, the approximation $\mathrm{Aj}$ and the detail $\mathrm{Dj}$ are built, considering the original signal as the approximation $\mathrm{A} 0$ at level 0 . Based on digital filters $h_{0}$ and $g_{0}$, a hierarchically

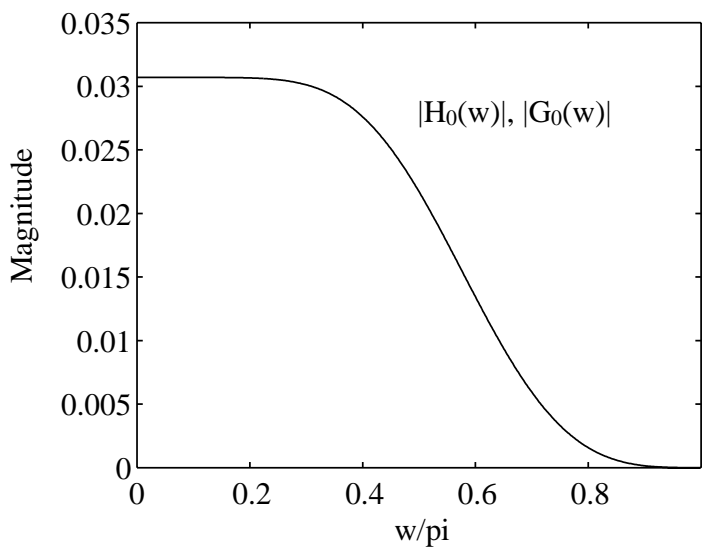

(c) Magnitude response

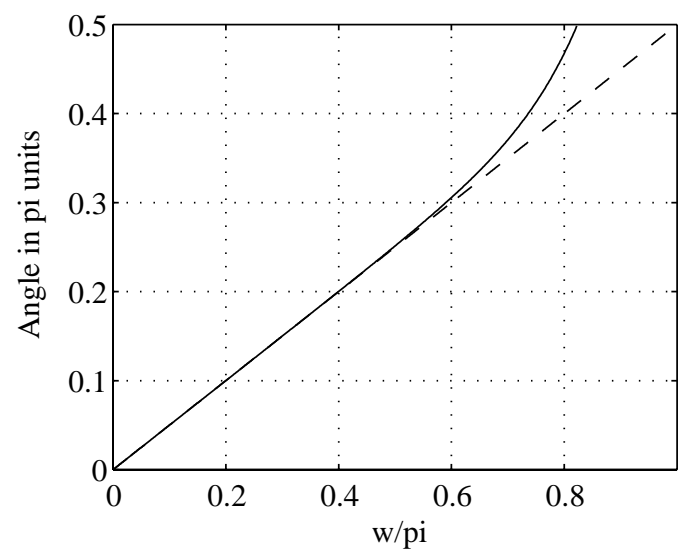

(d) Phase response

Fig. 1. (a) The digital filter $h_{0}(n)$; (b) A half-sample delayed version of $h_{0}(n)$; (c) The magnitude response of $h_{0}(n)$ and $g_{0}(n)$; (d) The phase response of $\boldsymbol{G}_{0}(\omega) / \boldsymbol{H}_{0}(\omega)$. 
structured decomposition of the shift-invariant DT DWT that is used to build an adaptive noise model of the background continuum for the application in a multipath environment, is illustrated in Figure 2. The degree of shift invariance of this scheme is examined and depicted in Figure 3 (a). The input is a unit step, shifted to 17

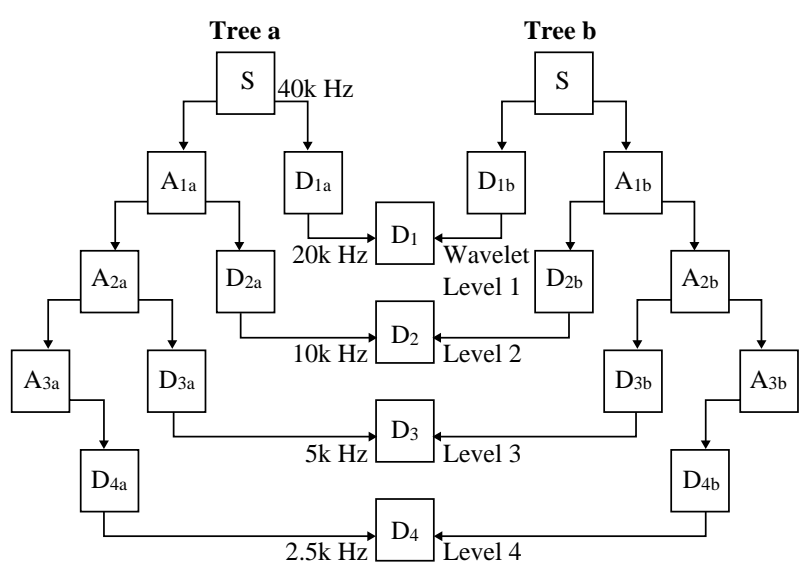

Fig. 2. Shift-invariant DT DWT.
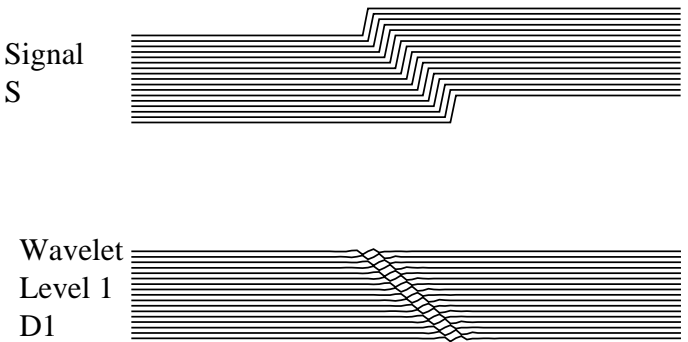

Level 2 D2

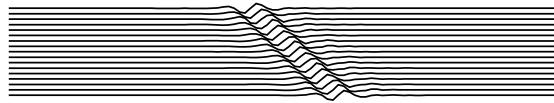

Level 3

D3

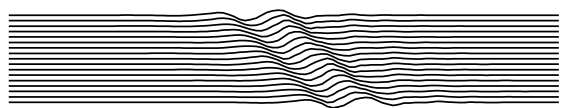

Level 4

D4

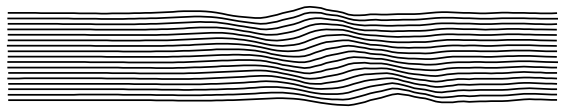

(a) DT DWT adjacent sampling instants in turn. Each unit step is passed through the shift-invariant DT DWT in Figure 2. The outputs D1, ..., D4 of this structure are reconstructed from the wavelet coefficients at each of levels 1 to 4 in turn and depicted in Figure 3 (a). A good shiftinvariant property is shown when all the 17 output components have the same shape for a given level. It is illustrated that the DT DWT in Figure 3 (a) is independent of shift while the imperfections of the DWT are fairly obvious in Figure 3 (b).

\section{SIGNAL DETECTION AND COMPUTER ANALYSIS}

According to [1], the discrete wavelet coefficients of underwater sound recordings can be divided into appropriate time scale, or time "window" that can be relevant to signal detection. Motivated by this application, this article considers the use of a shiftinvariant dual-tree discrete wavelet transform noise model to the problem of a multipath signal detection in underwater sound. The signal of interest is the dolphin sounds [5] denoted as $g(t)$. A multipath signal is produced from a linear combination of delayed replicas,

Signal

S

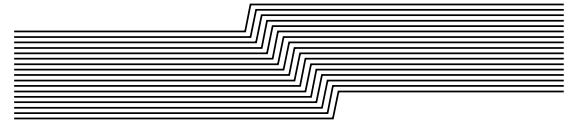

Wavelet

Level 1

D1

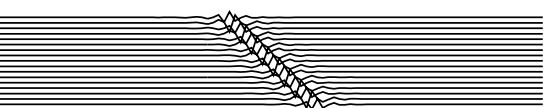

Level 2

D2

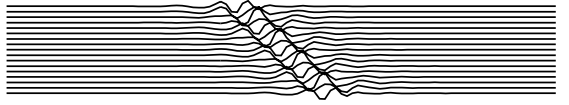

Level 3

D3

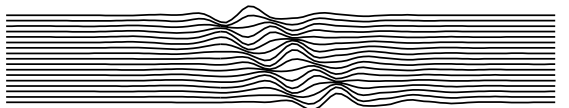

Level 4

D4

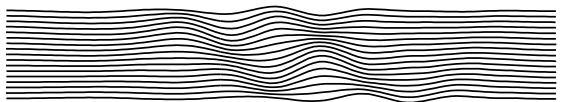

(b) DWT

Fig. 3. Wavelet components at level 1 to 4 of 17 shifted step responses of (a) DT DWT and (b) DWT. 


$$
g_{R}(t)=\sum_{m=1}^{M} \alpha_{m} g\left(t-\tau_{m}\right)
$$

where the number of replicas $M$, attenuation coefficients $\left\{\alpha_{m}\right\}$, and the delays $\left\{\tau_{m}\right\}$ are all unknown. Instead of wavelet coefficients, the reconstructing details of the dual-tree discrete wavelet transform of sound recordings are divided into appropriate time windows for detecting a multipath signal. In this section, the computer analysis will show that the good shift-invariant property of the proposed DT DWT noise model can improve the performance of a multipath signal detection, in a range of different signal-to-noise (SNR) ratios.

Figure 4 shows a time window divided from the shift-invariant DT DWT reconstructing details of recordings with a multipath dolphin sound [9]. The sound recordings are sampled at $44.1 \mathrm{kHz}$. This window containing 128 samples of the reconstructing detail (32768 data points) corresponds to a time period of 0.0029 seconds and is sufficient to capture significant changes of the dolphin sounds in our applications. The reconstructing details in level 1 to level 4 covering the frequency ranges that are of interest to us (i.e. $2-20 \mathrm{kHz}$ ) are summarized in terms of four mean sums of squares, $\left(x_{t, 1}, \ldots, x_{t, 4}\right)$ for each time window $t$,

$$
x_{t, j}=\frac{\sum_{k=1}^{128} D_{j, k}^{2}}{128}, j=1, \ldots, 4
$$

where $D_{j, k}^{2}$ are taken from a time window $t$. Because the signals of interest in this application are in the higher frequency range, these three mean sums of squares are used to form a vector of multivariate observation $\mathbf{x}_{t}=$ $\left(x_{t, 2}, \ldots, x_{t, 4}\right)$ in a time window $t$. The behavior of these
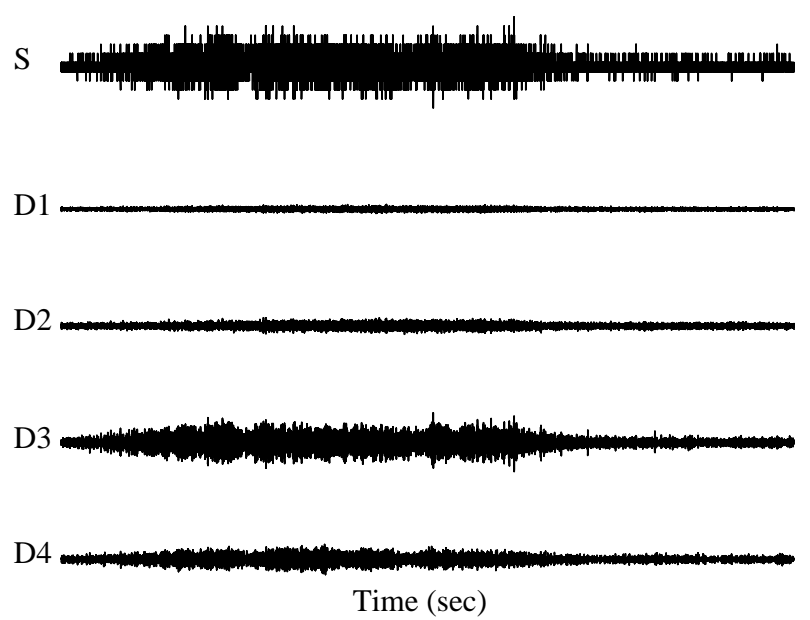

Fig. 4. Reconstructing details for a multipath dolphin sound using shift-invariant DT DWT. observations $\mathbf{x}_{t}$ covers the range of $2-10 \mathrm{kHz}$.

A behavior of observations during the appearance of a multipath dolphin sound is shown in Figure 5. Figure 6 illustrates its behavior during a period of noise when no "signals" are present. That the joint behavior of noise being significantly different from that of signals arrives at the same condition in [1]. This result suggests a method that uses density estimates of the shift-invariant DT DWT to establish the initial density estimate of noise and applies the detection criterion proposed by [1] to a shift-invariant DT DWT noise model to detect multipath signals. First, given data $\mathbf{x}_{1}$, $\ldots, \mathbf{x}_{T}$, the multivariate kernel density estimator [10] with a multivariate normal kernel is

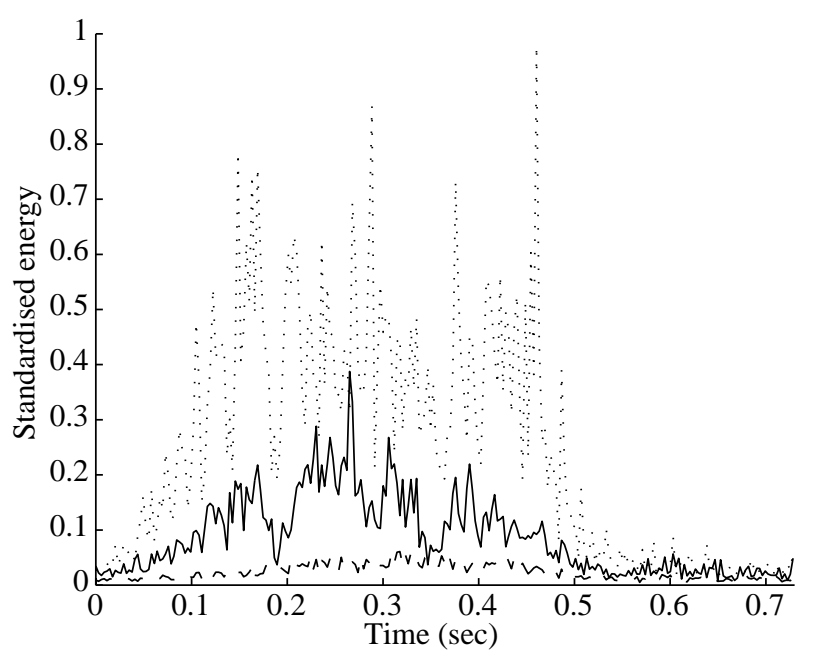

Fig. 5. Behavior of $x_{t}$ for a multipath dolphin sound ---, $x_{t, 2} ; \ldots, x_{t, 3}$; $-, x_{t, 4} \cdot$

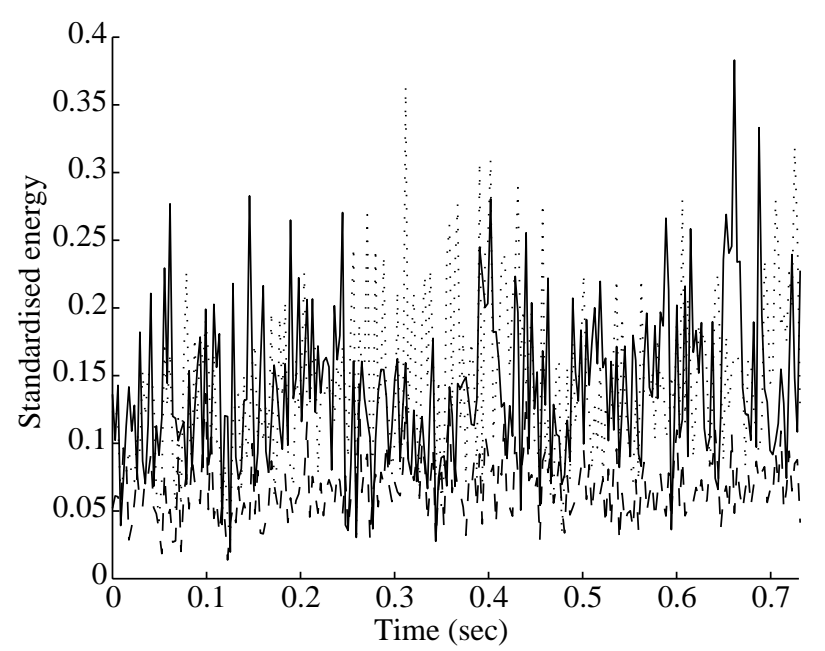

Fig. 6. Behavior of $x_{t}$ for background noise ---, $x_{t, 2} ; \ldots, x_{t, 3} ;---, x_{t, 4}$. 
Table 1. Performances of DT DWT and DWT signal detection methods

\begin{tabular}{ccccccc}
\hline & \multicolumn{2}{c}{ \% false alarm, $M=3$} & \multicolumn{2}{c}{ \% false alarm, $M=6$} & \multicolumn{2}{c}{ \% false alarm, $M=9$} \\
\hline SNR $(d B)$ & $D T D W T$ & $D W T$ & $D T D W T$ & $D W T$ & DT DWT & $D W T$ \\
\hline+5 & 0.2 & 1.6 & 0.2 & 0.7 & 0.5 & 1.2 \\
0 & 7.0 & 10.1 & 4.4 & 7.5 & 5.0 & 7.7 \\
-5 & 28.3 & 30.7 & 33.7 & 36.2 & 34.9 & 36.9 \\
-8 & 52.7 & 56.3 & 46.9 & 49.6 & 42.3 & 46.4 \\
-10 & 65.8 & 68.7 & 61.3 & 65.2 & 58.8 & 63.1 \\
\hline
\end{tabular}

Note: Comparison relates of identifying 35 known dolphin signals in 256 observations with noise at given SNRs and $M$.

$$
\hat{q}(\mathbf{x})=\frac{(\operatorname{det} \mathbf{H})^{1 / 2}}{(2 \pi)^{3 / 2} T h^{3}} \sum_{t=1}^{T} \exp \left(-\frac{\left(\mathbf{x}-\mathbf{x}_{t}\right)^{\prime} \mathbf{H}^{-1}\left(\mathbf{x}-\mathbf{x}_{t}\right)}{2 h^{2}}\right)
$$

where $\mathbf{H}$ is a robust estimate of the covariance matrix, and $h$ is a suitable global window width. Second, a detection criterion [1]

$$
\frac{\#\left\{\hat{q}_{t}\left(\mathbf{x}_{t}\right) \leq \hat{q}(\mathbf{x})\right\}+1}{T+1}
$$

is used to test if $\mathbf{x}$ is a signal or not, where "\#\{-\}" means "the number of" for $t=1, \ldots, T ; \hat{q}$ denotes the kernel density estimate based on $\mathbf{x}_{1}, \ldots, \mathbf{x}_{T} ; \hat{q}_{t}$ denotes the kernel density estimate based on $\mathbf{x}_{1}, \ldots, \mathbf{x}_{T}$ but excluding $\mathbf{x}_{t}$. Third, if this test produces a significantly small level, $\mathbf{x}$ is identified as an outlier from the current kernel density estimate and flagged as a potential signal and then subsequently ignored. Otherwise, this $\mathbf{x}$ will replace $\mathbf{x}_{T}$, $\mathbf{x}_{t}$ is replaced by $\mathbf{x}_{t+1}, t=1, \ldots, T-1$, and the optimal window width and the kernel density are updated before the next $\mathbf{x}$. Then a model from this method can be a baseline to identify the signals at any time. Examining the results when this proposed method is applied to a multipath signal $g_{R}(t)$ in (1), the number of paths $M$ is first set to 3. All attenuation factors $\left\{\alpha_{m}\right\}$ are equal to 1 , and the set of delay times $\left\{\tau_{m}, m=1,2,3\right\}$ are generated by a random number generator in each trial, to produce a multipath signal $g_{R}(t)$. Using different SNRs to produce a range of test datasets. Each test dataset is designed to provide 512 successive multivariate observations, $\mathbf{x}_{t}=\left(x_{t, 2}, \ldots, x_{t, 4}\right)$. The first 256 observations are guaranteed a priori to contain no signals. For the following 256 observations, 221 are known to relate only to noise, 35 are related to a multipath signal with noise superimposed. It is immediately processed on the first 256 observations to provide an initial stable shiftinvariant DT DWT noise model. Successive 256 observations are then considered. As a result, each of the corresponding 256 observations is detected as a signal or as background noise.
Table 1 summaries the results obtained over the various test datasets at given $M$ and SNRs of the 100 trials, where "false alarm" refers to the average of the percentage of misclassifications when a signal is classified as noise. For example, one of the results that obtained over the test datasets at given $M=6$ and SNR $=-8 \mathrm{~dB}$ of the 100 trials, shows that the value of $46.9 \%$ of the false alarm probability of DT DWT is less than that value of $49.6 \%$ of DWT. It shows that this method can increase the performance of a multipath signal detection, by reducing the false alarm probability in a range of different SNR ratios.

Figure 7 is a plot of the DWT reconstruction (top) and the DWT detection results (Bottom) of a multipath dolphin sound $g_{R}(t)$ at $\mathrm{SNR}=0 \mathrm{~dB}$ and $M=6$. Figure 8 is a plot of the DT DWT reconstruction (top) and the DT DWT detection results (Bottom) of a multipath dolphin sound $g_{R}(t)$ at the same condition. Under the same $M=$ 6 , but now set SNR $=-8 \mathrm{~dB}$, the DWT reconstruction and its detection results of a multipath dolphin sound is plotted in Figure 9, while the DT DWT reconstruction and its detection results of the same signal is plotted in

$\mathrm{D}_{2}$

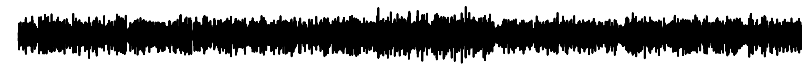

$\mathrm{D}_{3}$

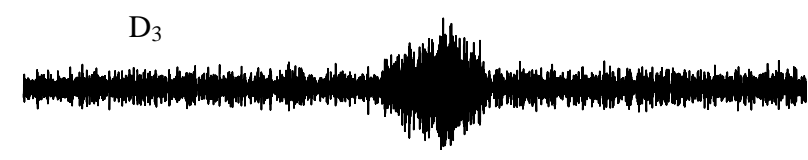

$\mathrm{D}_{4}$

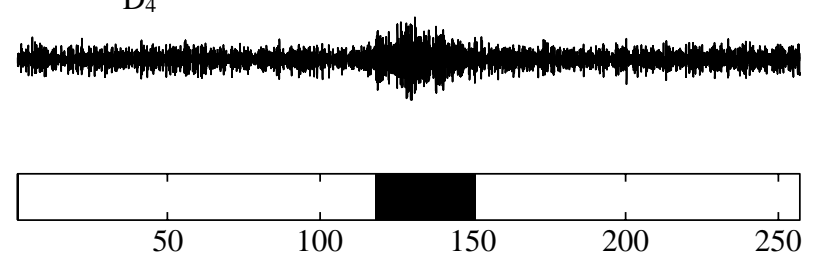

Fig. 7. The DWT reconstruction (Top) and its detection results (Bottom) of a multipath dolphin sound at $\mathrm{SNR}=0 \mathrm{~dB}$ and $M=6$. 
$\mathrm{D}_{2}$

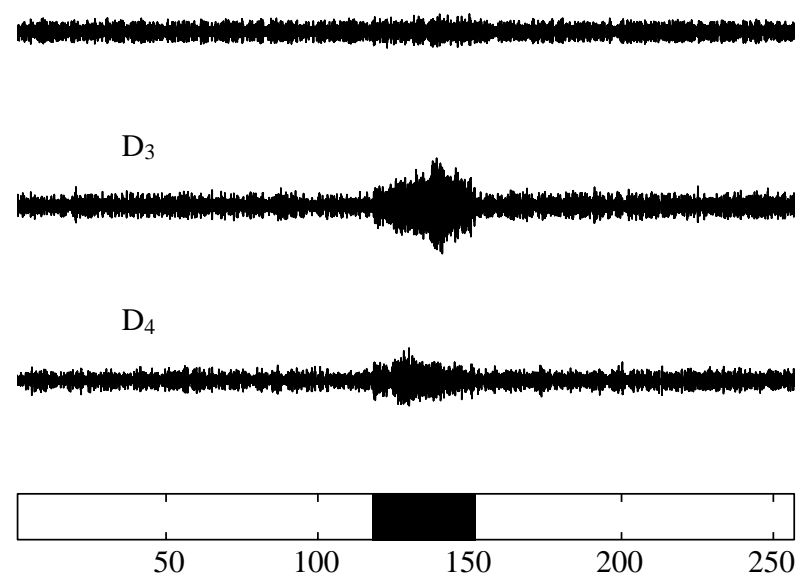

Fig. 8. The DT DWT reconstruction (Top) and its detection results (Bottom) of a multipath dolphin sound at SNR $=0 \mathrm{~dB}$ and $M=6$.

$\mathrm{D}_{2}$

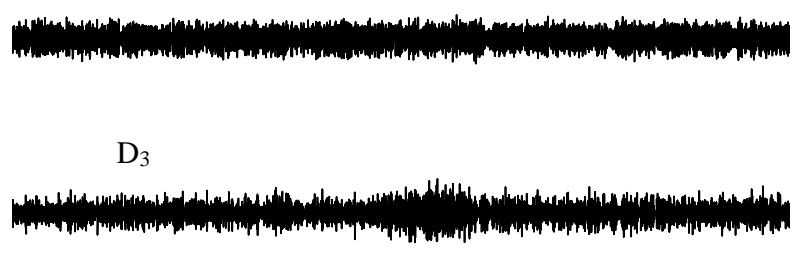

$\mathrm{D}_{4}$

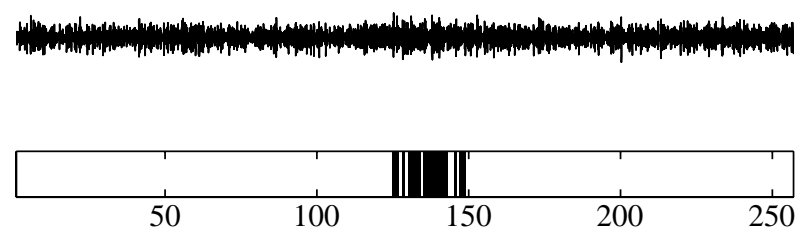

Fig. 9. The DWT reconstruction (Top) and its detection results (Bottom) of a multipath dolphin sound at $\mathrm{SNR}=-8 \mathrm{~dB}$ and $M=6$.

Figure 10. Finally, setting SNR $=-10 \mathrm{~dB}$ with the same $M=6$, the DWT and DT DWT's detection results are shown in Figure 11 and Figure 12, respectively. These plots illustrate that the shift-invariant DT DWT noise model has better detecting performance of a multipath signal than its counterpart at different SNRs.

\section{CONCLUSION}

This paper proposes the use of a shift-invariant DT DWT noise model to the problem of a multipath signal detection in underwater sound. When the input signal is shifted as in a multipath environment, this shift-invariant DT DWT can generate multi-resolution subspaces that keep more of their coefficient energy in each of these subspaces then DWT's. The result of computer
$\mathrm{D}_{2}$
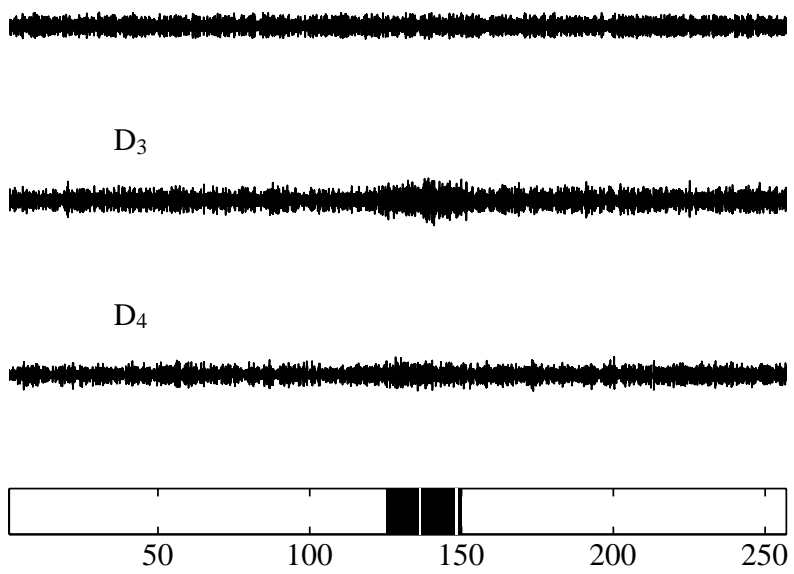

Fig. 10. The DT DWT reconstruction (Top) and its detection results (Bottom) of a multipath dolphin sound at $\mathrm{SNR}=-8 \mathrm{~dB}$ and $M=6$.

$\mathrm{D}_{2}$

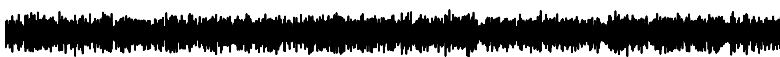

$\mathrm{D}_{3}$

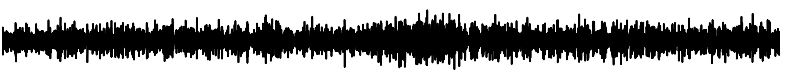

$\mathrm{D}_{4}$
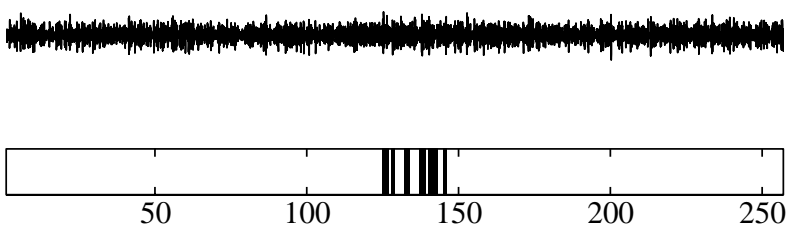

Fig. 11. The DWT reconstruction (Top) and its detection results (Bottom) of a multipath dolphin sound at $\mathrm{SNR}=-\mathbf{1 0} \mathrm{dB}$ and $M=6$.

$\mathrm{D}_{2}$

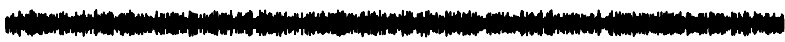

$\mathrm{D}_{3}$

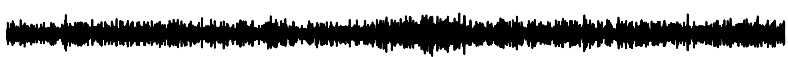

$\mathrm{D}_{4}$

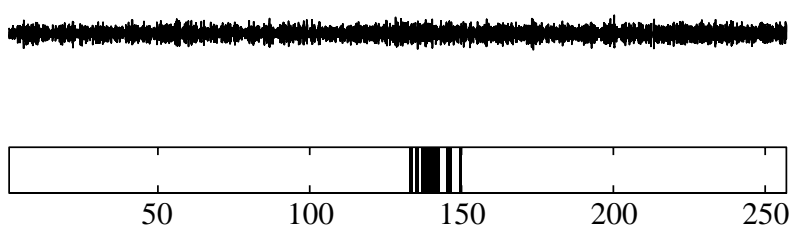

Fig. 12. The DT DWT reconstruction (Top) and its detection results (Bottom) of a multipath dolphin sound at $\mathrm{SNR}=-\mathbf{- 1 0 \mathrm { dB }}$ and $M=6$. 
simulations shows that the proposed method can improve the performance of a multipath signal detection, by reducing the false alarm probability in a range of different SNR ratios. For example, one of the results that obtained over the test datasets at given $M=3$ and SNR $=-8 \mathrm{~dB}$ of the 100 trials, shows that the value of $52.7 \%$ of the false alarm probability of DT DWT is less than that value of $56.3 \%$ of DWT.

\section{ACKNOWLEDGEMENTS}

This work was supported by the National Science Council of the R.O.C. under contract NSC 93-2611-E019-024.

\section{REFERENCES}

1. Bailey, T.C., Sapatinas, T., Powell, K.J., and Krzanowski, W.J., "Signal Detection in Underwater Sound Using Wavelets," Journal of the American Statistical Association, Vol. 93, No. 441, pp. 73-83 (1998).

2. Benno, S.A. and Moura, J.M.F., "On Translation Invariant Subspaces and Critically Sampled Wavelet Transforms," Multidimensional Systems and Signal Process., Vol. 8, pp. 89-110 (1997).
3. Chang, S.H. and Wang, F.T., "Application of the Robust Discrete Wavelet Transform to Signal Detection in Underwater Sound," International Journal of Electronics, Vol. 90, No. 6, pp. 361-371 (2003).

4. Daubechies, I., "Orthonormal Bases of Compactly Supported Wavelets," Communications on Pure and Applied Mathematics, Vol. 41, pp. 909-996 (1988).

5. Goodall, M., The Way of the Ocean, New World Music, Switzerland (1998).

6. Kingsbury , N.G., "Complex Wavelets for Shift Invariant Analysis and Filtering of Signals," Applied and Computational Harmonic Analysis, Vol. 10, No. 3, pp. 234-253 (2001).

7. Mallat, S.G., "A Theory for Multiresolution Signal Decomposition: the Wavelet Representation," IEEE Transactions on Pattern Recognition and Machine Intelligence, Vol. 11, pp. 674-693 (1989).

8. Selesnick, I.W., "Hilbert Transform Pairs of Wavelet Bases," IEEE Signal Processing Letter, Vol. 8, pp. 170173 (2001).

9. Selesnick, I.W., "The Design of Approximate Hilbert Transform Pairs of Wavelet Bases," IEEE Transactions of Signal Processing, Vol. 50, pp. 1144-1152 (2002).

10. Silverman, B.W., Density Estimation for Statistics and Data Analysis, Chapman and Hall, London, p. 78 (1986). 